\title{
CNG FUEL LEAKAGE DETECTION AS EMISSIONS PREVENTION
}

\author{
Rafael Amend da Cruz and Frederico Tischer
}

Robert Bosch Ltda

Emails: rafael.cruz@br.bosch.com and frederico.tischer@br.bosch.com

\begin{abstract}
The paper describes and presents practical results of a method for detection of internal fuel leakage in an engine. The method is based in unexpected oxygen concentration changes measured in the exhaust gases together with the Engine Roughness (disturbance on the Low Idle controller). The concept is useful to detect small leakages or a fuel injector blocked in the open position.
\end{abstract}

This strategy can be used when the fuel injectors are blocked in the open position and the engine is in one of these two states: no external torque demand and torque is controlled by the low idle controller (Low Idle) or no external torque demand and torque is increasing due to the drag of the engine external influence (Overrun). The measurements were performed on a system with natural gas injection in a Dual Fuel (Diesel + CNG) vehicle equipped with a lambda sensor on the exhaust pipe.

The measurements were performed on a system with natural gas injection in a Dual Fuel vehicle.

\section{Introduction}

With growing concern about emissions of GHG (greenhouse gases) the use of natural gas as fuel has been seen as a very interesting alternative. Natural gas, whose main constituent is methane, due to its higher ratio of hydrogen / carbon and their higher content of energy generates about $22 \%$ less carbon dioxide $\left(\mathrm{CO}_{2}\right)$ emissions compared to diesel considering the same combustion efficiency. Considering raw emissions, the reduction in the particulates emission is very significant which allows a calibration focused on reduction of $\mathrm{NO}_{\mathrm{x}}$. The $\mathrm{HC}$ emissions are higher than those of a typical diesel engine and consist primarily of $\mathrm{CH}_{4}$ (methane).

Dual fuel engine performance and emissions vary depending on operating conditions and the sophistication of the control system. Dual fuel engines perform best under moderate to high load, and can often be equal or better regarding the fuel-efficiency of a pure diesel engine under the same conditions. Operating with a lean air-fuel ratio, the dual fuel engines can also achieve much lower emissions (especially of NOx and particulate matter (PM)) than a pure diesel. Existing dual fuel conversions suffer from major increases in carbon monoxide $(\mathrm{CO})$ and hydrocarbon $(\mathrm{HC})$ 
emissions and loss of fuel efficiency at light loads. This is because they operate unthrottled, so that the air-fuel mixture becomes leaner as the load is reduced. As the mixture becomes leaner, combustion eventually degrades, leaving large amounts of partial reaction products in the exhaust.

Therefore, among the main challenges regarding the usage of CNG as a fuel on internal combustion engines is to keep the levels of $\mathrm{CH}_{4}$ (methane) emissions according to international standards for emissions legislation. Consequently, there is an increasing necessity for systems where the emission relevant components are well controlled and any malfunction of those is detectable in order to avoid the release of the CNG in an undesired manner.

\section{Internal Leakage Diagnosis}

The diagnosis method presented on this paper was developed for a Dual Fuel system in order to detect if there is a leakage through the CNG valves when they are not energized.

\section{Introduction to the Method}

On some operation modes the unexpected injection of CNG leads to an increase in the level of emissions to the environment, therefore the method presented on this paper was introduced to guarantee that the system emissions level will not be influenced by the unexpected CNG injection.

\subsection{Oxygen concentration Sensor}

The technique used on this method consists in measuring the oxygen concentration level on the exhaust pipes of the system and to measure this concentration a sensor (Lambda probe) is used. The sensor does not actually measure oxygen concentration, but rather the difference between the amount of oxygen in the exhaust gas and the amount of oxygen in the air by a given air-fuel ratio, which is the ratio between the mass of air and the mass of fuel in the fuel-air mix at any given moment.

This sensor is a (solid-state electrolyte made of ceramic material. At high temperatures, this electrolyte becomes conductive and generates a characteristic galvanic charge at the sensor connections; this voltage is an index of the gas oxygen content [1]). Rich mixture causes an oxygen demand. This demand causes a voltage to build up, due to transportation of oxygen ions through the sensor layer. Lean mixture causes low voltage, since there is an oxygen excess. The sensor element is a ceramic cylinder plated inside and out with porous platinum electrodes; the whole assembly is protected by metal gauze. It operates by measuring the difference in oxygen between the exhaust gas and the external air, and generates a voltage or changes its resistance depending on the difference between the two.

The sensors only work effectively when heated to approximately $600{ }^{\circ} \mathrm{C}$ (on this case it was used to validate the method develop a Lambda sensor with 
the temperature of approximately $600{ }^{\circ} \mathrm{C}$ ), so most newer lambda probes have heating elements encased in the ceramic that bring the ceramic tip up to temperature quickly. The length of time required to bring the probe to temperature depends on the temperature of the ambient air and the geometry of the exhaust system.

The exhaust gas flowing through the lambda sensor will be subjected to the high temperatures of the environment inside of the lambda sensor and, with the presence of oxygen, the hydrocarbons present on these gases will be oxidized. This oxidization will lead to a reduction of the oxygen concentration on the exhaust pipe translated by the lambda sensor into electrical signal interpreted by the ECU of the engine.

The method for leakage detection is applied in two operation modes of the Dual Fuel engine: Low Idle and Overrun.

The concept is that on these engine operation modes, the lambda sensor has characteristic values from the normal operation without CNG injection. In the case of the Overrun condition the lambda value on the system goes up to a value of infinite because there is nothing but air passing through the exhaust pipe. On the Low Idle condition the expected lambda value can be calculated via modeling with the characteristics of the engine due to the Diesel injection demanded to keep the engine running.

To perform the diagnostics the actual lambda value measured by the sensor is compared to the expected value. Additionally, during a Low Idle condition, the engine roughness can be monitored according to the variation of the engine speed.

If, by any unforeseen cause, there is CNG being injected or leaked during one of these two modes, this CNG will influence the measured lambda value reducing the oxygen concentration in comparison to the expected value. If this happens, the system can detect and perform a reaction measure (for Dual Fuel system, for example, it's possible to change to Diesel mode and close the CNG tank valves this will be informed to the driver by lighting up the MIL) in order to minimize fuel leakage into the environment and consequently reduce the level of emissions.

\section{2. $\mathrm{CNG}$ as emission}

CNG combustion produces fewer undesirable gases than the other common used fuels. It is safer than other fuels in the event of a leak, because natural gas is lighter than air and disperses quickly when released. CNG is made by compressing natural gas (which is mainly composed of methane, $\mathrm{CH}_{4}$ ). It is stored and distributed in hard containers with a pressure range of 200 to $248 \mathrm{bar}$.

Natural gas produces lower carbon dioxide $\left(\mathrm{CO}_{2}\right)$ emissions compared to other fuels. While CNG vehicles do emit methane, one of the main greenhouse gases, any increase in methane emissions is more than offset by a substantial reduction in $\mathrm{CO}_{2}$ 
emissions in respect to other fuels. On the other hand, (the contribution of the methane to the greenhouse gases is 25 times larger than the contribution of $\mathrm{CO}_{2}$ [2]).

Although this typical emission of methane, which is inherent of the CNG fuelled systems, the use of CNG still bring benefits regarding greenhouse gases.

The potential to reduce emissions also depends on the basic diesel engine. Modern common rail engines have a higher potential due to the variety of parameters that are controlled by the electronic engine management. However this requires a profound knowledge of the OEM's engine control, an obstacle for retrofit applications. With the availability of today's new systems that can work without interfering the OEM control unit, this problem may be overcome.

Natural gas in combustion engines is a very clean fuel. It is free of particulate matters (PM) and produces less $\mathrm{CO}_{2}$ than petrol fuel. The emissions of dual fuel engines therefore show less PM and $\mathrm{CO}_{2}$, the amount of reduction is depending on the share of CNG added, the type of the basic diesel engine and the engine control management.

Typically assuming a $50 \%$ content of CNG, the reduction of $\mathrm{CO}_{2}$ is between $10-15 \%$, PM reduction is up to $60 \%$. This information is described on the figure 1 below [3].

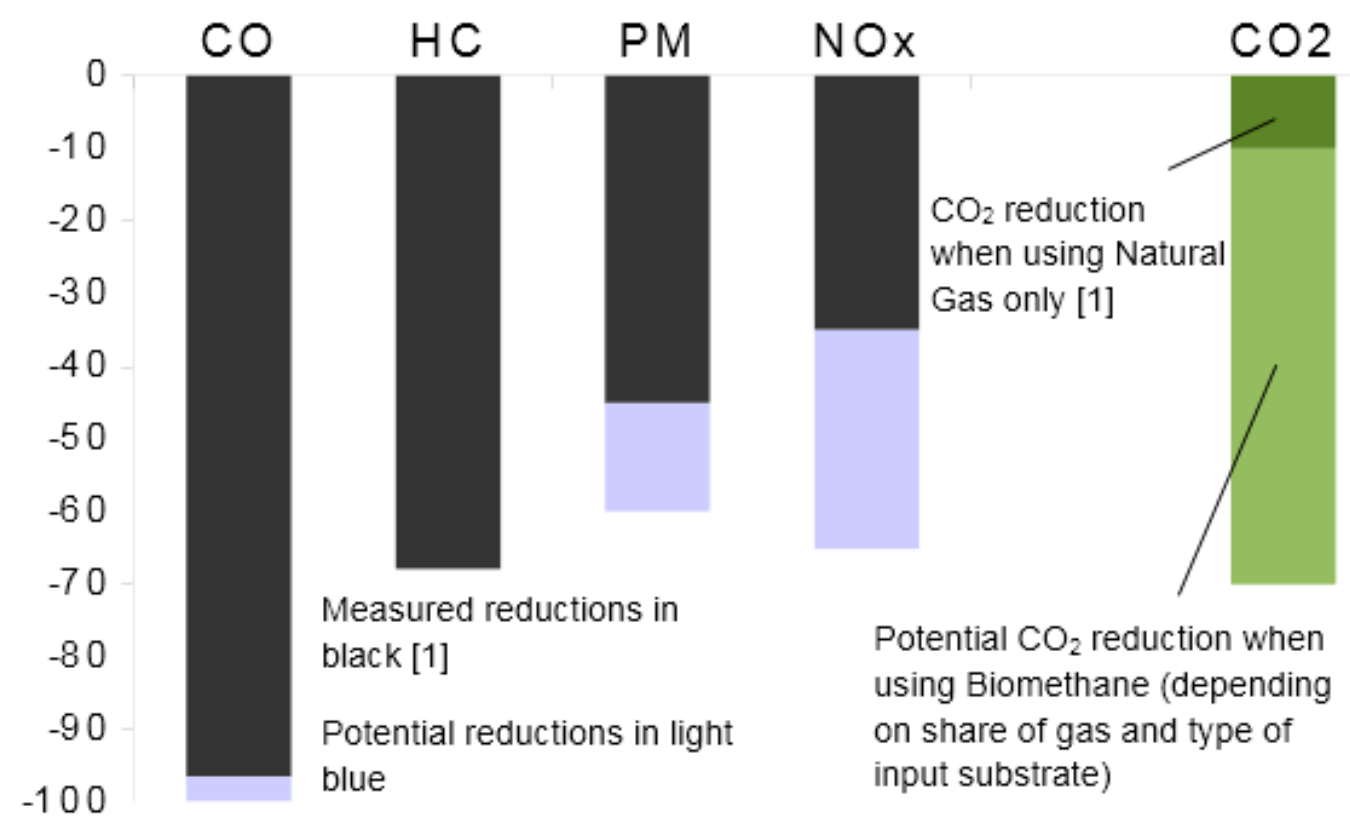

Figure 1: Reduction of Emissions for Dual Fuel engine

\section{System used for testing}

A diesel and natural gas system is called Dual-Fuel system as both fuels are needed for an optimal combustion. 
The Dual-Fuel system is based on using a pilot diesel fuel injection as a substitute for the sparkle plug from Otto engines and the natural gas flows into the engine with the air together through the intake manifold.

In the intake stroke the natural gas mixed with the air stream flows into the cylinder through the intake ports. At the compression stroke the mixture is compressed resulting in temperature and pressure increasing, which allows the diesel pilot injection self-ignition. The most critical during this stroke is the control of knocking once the diesel compression rate is maintained. At the power stroke the diesel combustion ignites the natural gas, rising up fast the temperature and pressure, forcing the piston downwards. At the exhaust stroke the burned gas leave the cylinder through the exhaust port. On the figure 2 is possible to see a layout of a Dual Fuel system with single point injection (all CNG injectors injecting on the same point of the intake manifold).

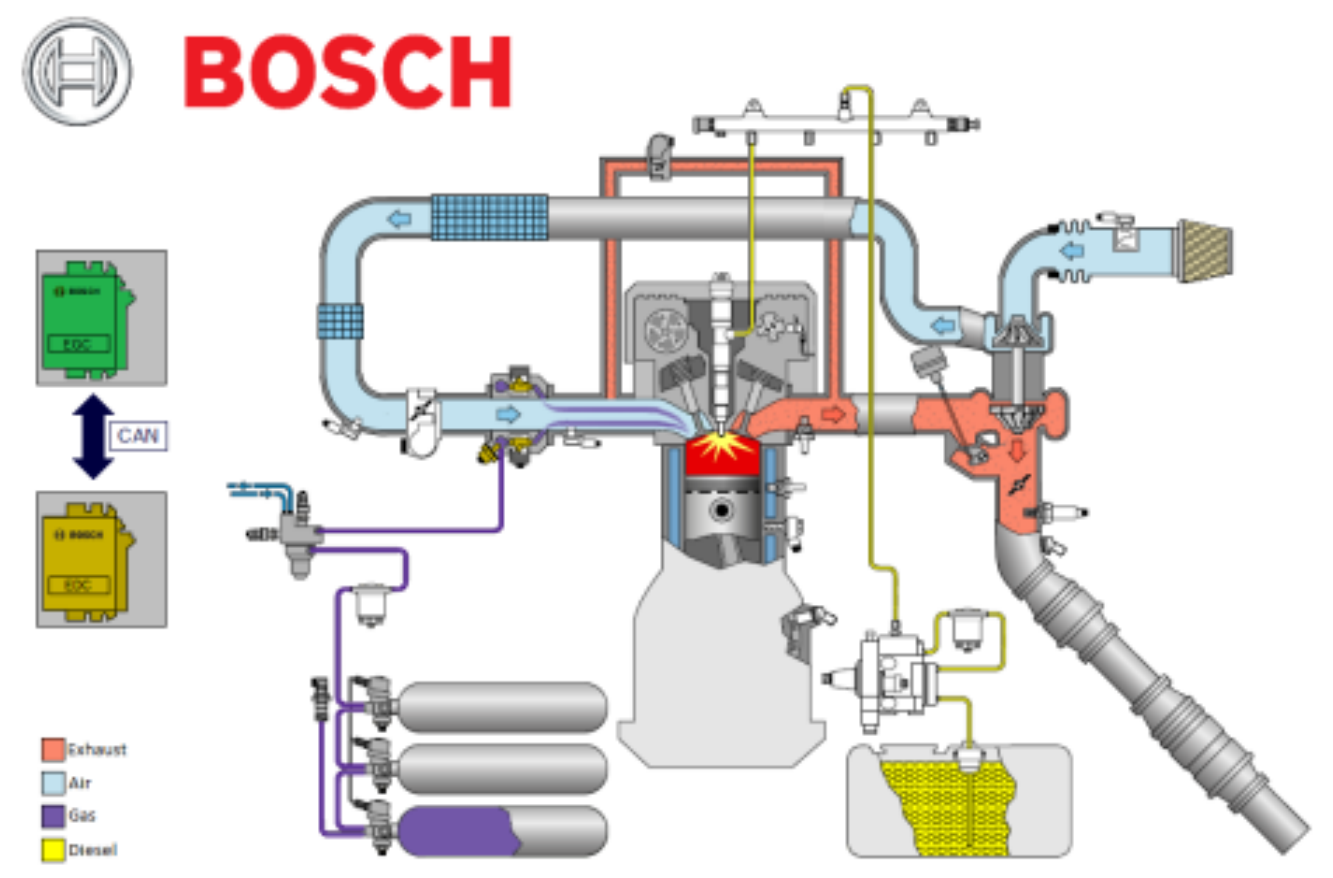

Figure 2: Layout of a Dual Fuel System

\section{Leakage detection tests}

For the development of the function to detect the leakage some tests were performed on the test bench to validate the concept of the function and to measure the level of leakage that can be detected during the Low Idle condition.

The procedure that was used consisted on creating a controlled leakage on the system simulating a Injector Leakage (internal leakage) and measuring the Lambda value (Oxygen concentration) with a sensor and the Engine Speed. Additionally, it was calculated the expected value of Lambda based on the ratio between Air and Fuel Quantity. 
By performing this procedure, it was possible to conclude that, even for low leakage levels, the engine speed show some disturbance on the Low Idle controller and the expected Lambda value is presenting a significant deviation from the measured Lambda value.

The figure 3 presents the results achieved on these tests. It consists of four steps of leakage level, as described below:

- First Step (-10 to 0 s) - No leakage: engine speed stable at $600 \mathrm{rpm}$ and expected lambda value matching measured value. Usual consumption of fuel is $7 \mathrm{~kg} / \mathrm{h}$.

- Second Step (0 to $8 \mathrm{~s}$ ): $0.6 \mathrm{~kg} / \mathrm{h}$ leakage.

- Third Step (8 to $18 \mathrm{~s}$ ): $1.1 \mathrm{~kg} / \mathrm{h}$ leakage.

- Fourth Step (18 to $26 \mathrm{~s}$ ): $1.7 \mathrm{~kg} / \mathrm{h}$ leakage.

Based on the results it is possible to see that detecting leakage is possible due to the disturbances that can be observed on the expected lambda values and, in addition, the engine speed can also be used due to disturbs on Low Idle controller.

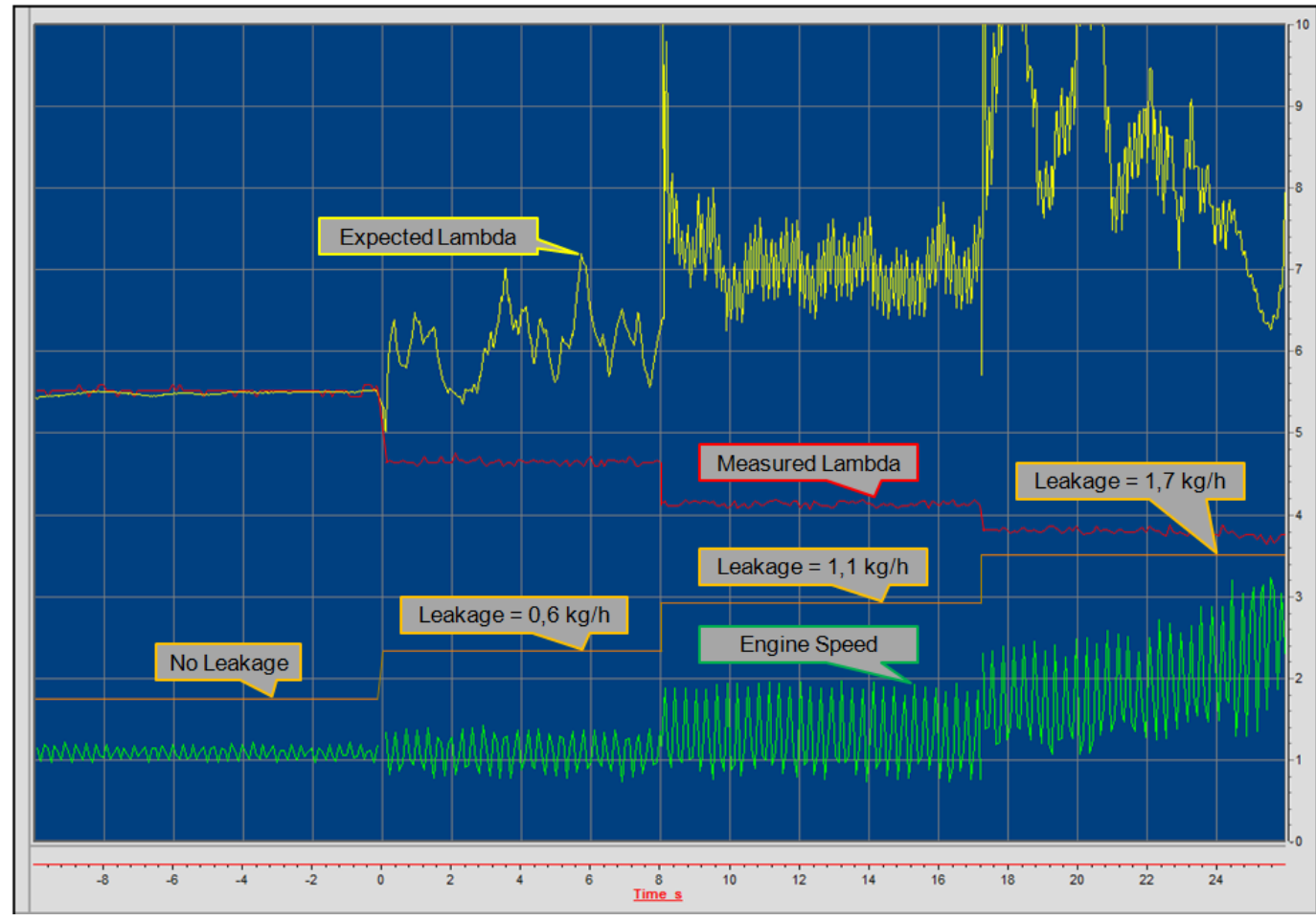

Figure 3: Threshold for leakage detection - Low Idle

\section{Leakage detection tests - validation}

The typical behavior of the lambda value in Low Idle in the Dual Fuel system used for the measurements can be seen on the figure 4 . 


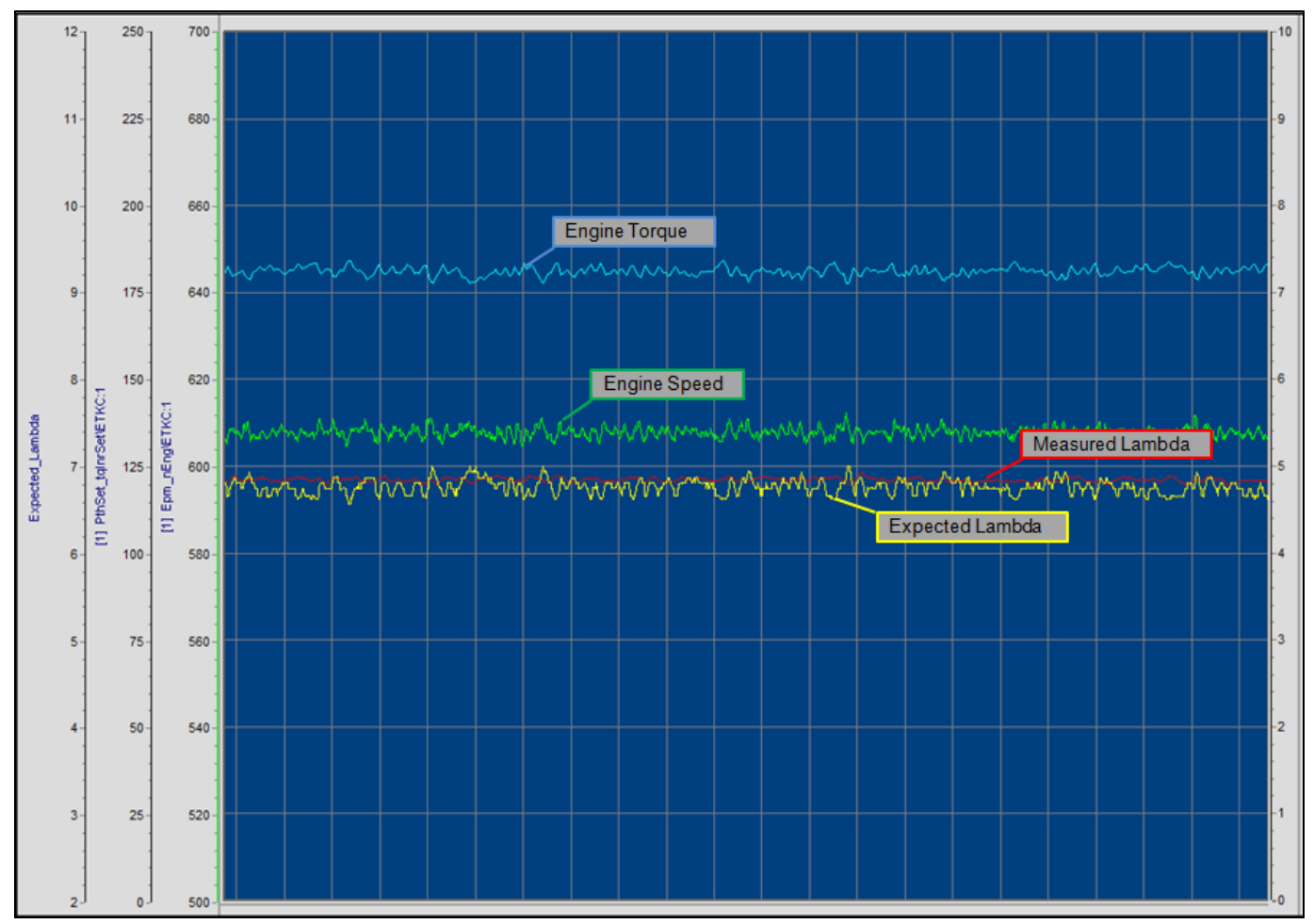

Figure 4: Typical Lambda values for Low Idle condition

In the figure 5 it can be observed the behaviour of the system when under the influence of a leakage considering that one CNG injector is open at all times. In an attempt to keep the low idle speed, the system reduces the Diesel quantity in a way that leads to a very high expected lambda value and due to misfire a disturbance on the engine speed (engine roughness) can be also noticed.

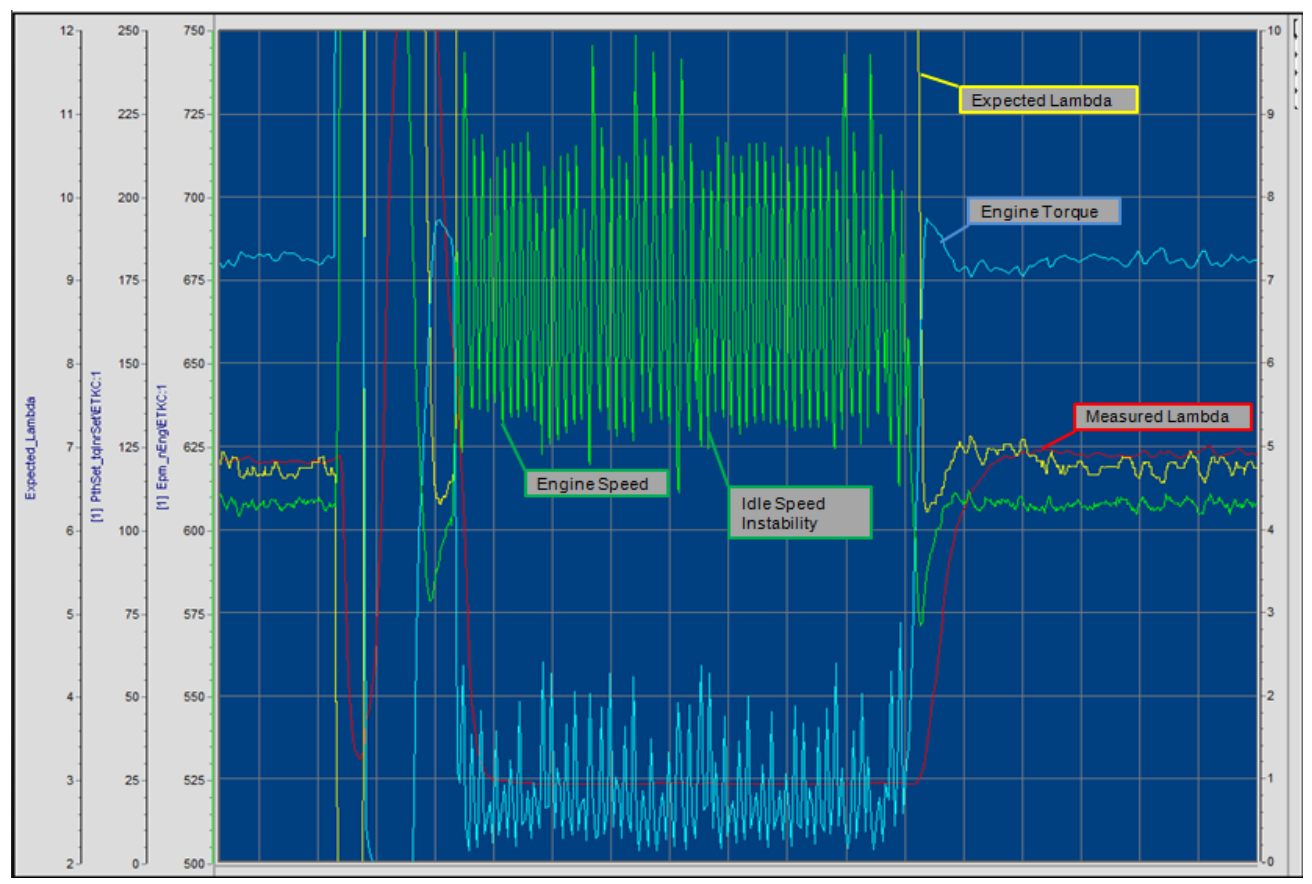

Figure 5: System under leakage influence - Low Idle condition 


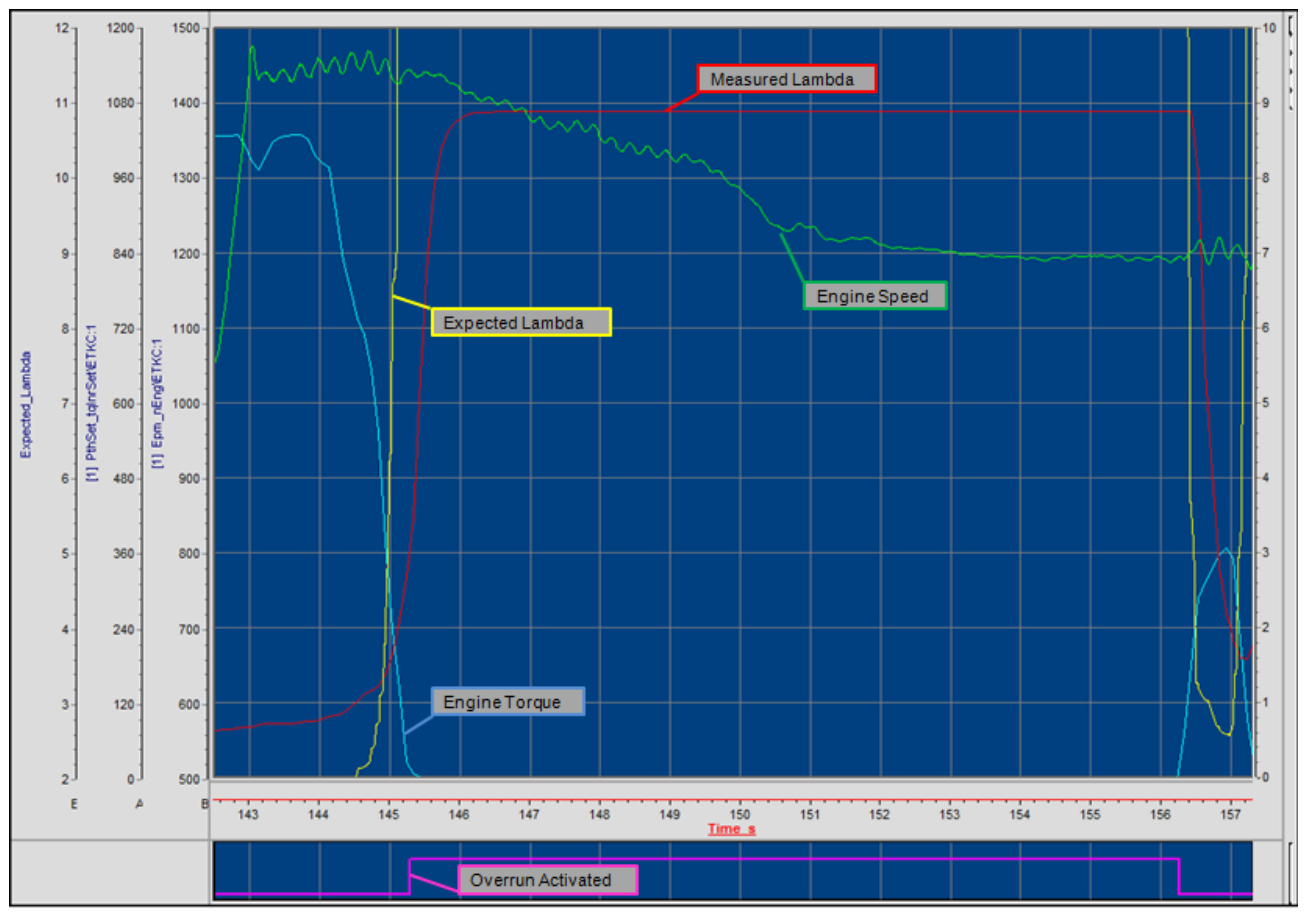

Figure 6: Typical Lambda values for Overrun condition

The typical behaviour of the lambda value in Overrun condition in the Dual Fuel system used for the measurements can be seen on the figure 6 . During the Overrun the systems stops to inject fuel and, for this reason, the expected lambda value goes to infinite and the measured lambda goes to the end of the scale (16).

In the figure 7 it can be observed the behaviour of the system when under the influence of a leakage considering that one CNG injector is open from the time $118,2 \mathrm{~s}$ of the measurement on. During the Overrun after the opening of the valve simulating the leakage, the measured lambda value goes down to a value of 1.4 due to the unexpected CNG being injected. 


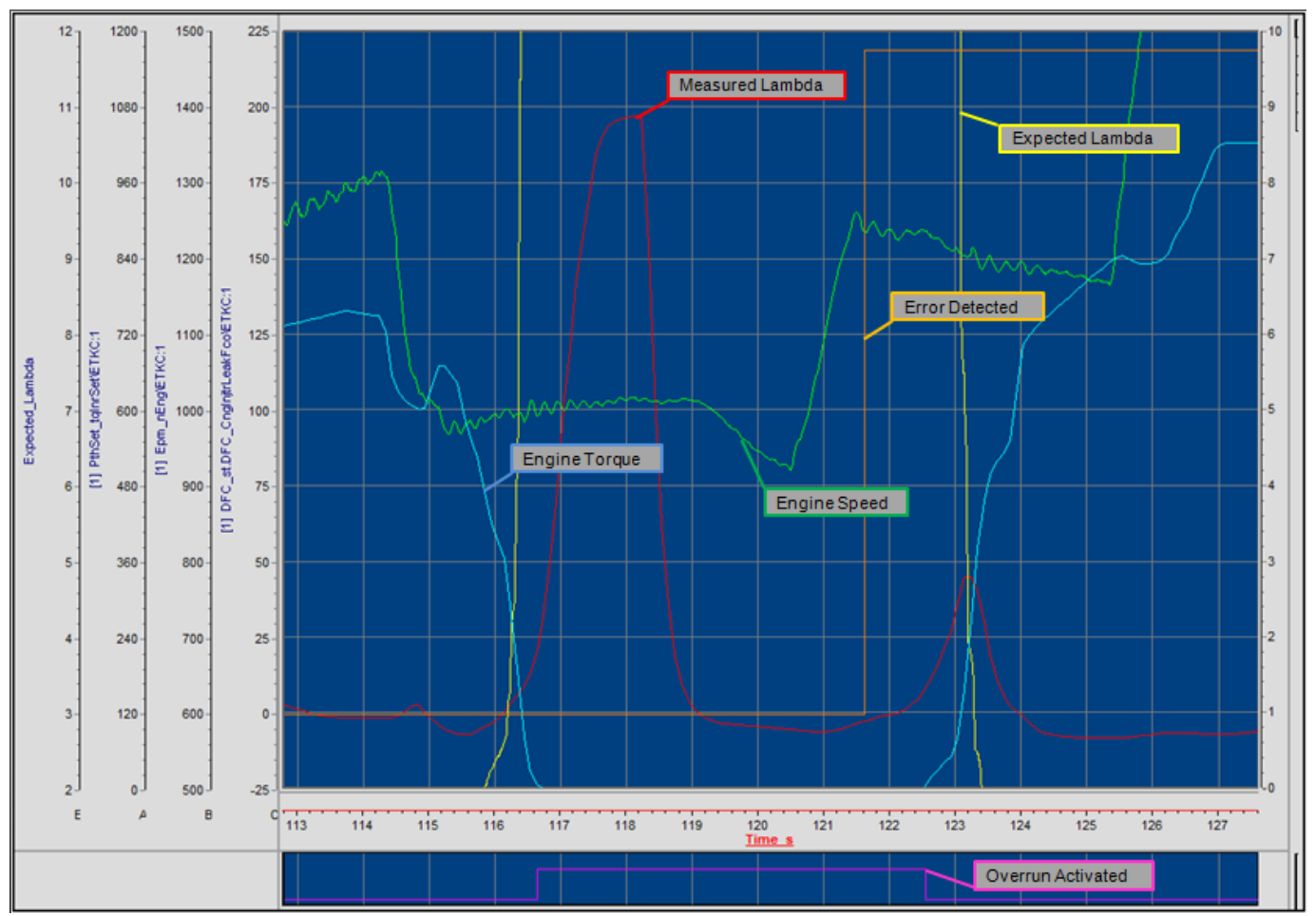

Figure 7: System under leakage influence - Overrun condition

In the figure 7 it is possible to see the debounce time of 4 seconds for identify the error on the system.

\section{Conclusion}

Using the measurements performed on the Dual Fuel vehicle it is possible to notice the feasibility of the use of a strategy to detect fuel leakage using the oxygen concentration level.

Additionally to the positive environmental impact of the use of this method it is important to keep in mind that with the fast detection and reaction to this leakage it is also prevented a degradation of the catalyst that would lead to further environmental impacts due to higher emissions.

Although the tests were performed on an engine using a Dual Fuel injection system, the concept of the method is also applicable for other kind of engines (gasoline or diesel).

\section{References}

[1] BOSCH, Robert. Manual de Tecnologia Automotiva. Local: Edgard Blücher, 2005. 
[2] INTERGOVERNMENTAL PANEL ON CLIMATE CHANGES. Climate Change 2007: The Physical Science Basis. Local: Cambridge, 2008.

[3] BRACHETTI, Jürgen. Dual Fuel - The best fuel in the most efficient engine. Local: Kaiserslautern, May, 2010. 